\title{
$A b$ initio calculation of ionization potential and electron affinity of six common explosive compounds
}

This article was published in the following Dove Press journal:

Reports in Theoretical Chemistry

12 November 2012

Number of times this article has been viewed

\author{
Jason K Cooper' \\ Christian D Grant ${ }^{2}$ \\ Jin Z Zhang'
}

'Department of Chemistry and Biochemistry, University of California, Santa Cruz, CA, ${ }^{2}$ Physical and Life Sciences, Chemical Sciences Division, Lawrence Livermore National Laboratory, Livermore, CA, USA
Correspondence: Jin Z Zhang Department of Chemistry and Biochemistry, University of California, Santa Cruz, CA 95064, USA

Email zhang@ucsc.edu

Christian D Grant

Physical and Life Sciences

Chemical Sciences Division

Lawrence Livermore National

Laboratory, 7000 East Ave,

Livermore, CA 94550, USA

Email grant29@IInl.gov
Abstract: The vertical and adiabatic ionization potential $\left(\mathrm{IP}_{\mathrm{V}}\right.$ and $\left.\mathrm{IP}_{\mathrm{A}}\right)$ and vertical electron affinity (EA $A_{v}$ for six explosives, hexogen (RDX), octogen (HMX), triacetone triperoxide (TATP), hexamethylene triperoxide diamine (HMTD), 2,4,6-trinitrotoluene (TNT), and pentaerythritol tetranitrate (PETN), have been studied using ab initio computational methods. The IP was calculated using MP2, CBS-QB3, and Koopmans' theory, while the $\mathrm{IP}_{\mathrm{A}}$ was calculated with B3LYP, CAM-B3LYP, $\omega B$ 97XD, B2PLYP, and MP2 using the $\triangle \mathrm{E}$ method for the ground state B3LYP optimized geometries. IP $\mathrm{A}$ of RDX and TNT were also calculated using CBS-QB3 with relaxed geometries of the ions. Of the methods tested, B3LYP and B2PLYPD provided superior and more consistent results for calculating the IP compared to CBS-QB3 level $\mathrm{IP}_{\mathrm{A}}$ calculations and experimental data (where available). CBS-QB3 was used as a benchmark for calculating the $\mathrm{EA}_{\vee}$ as experimental data has not been reported. For calculations of the EA, B3LYP performed the worst while MP2 and B2PLYPD predicted values closest to those made by CBS-QB3. Basis set effects were evaluated using 6-31+G(d,p), 6-311+G(d,p), and 6-311+G(3df,2p) for both IP and EA. $6-31+\mathrm{G}(\mathrm{d}, \mathrm{p})$ gave satisfactory results for calculating IP while $6-311+\mathrm{G}(3 \mathrm{df}, 2 \mathrm{p})$ had improved results for calculating the EA. The four nitro-containing compounds have exothermic reduction potentials while the peroxides are endothermic. In addition, it was determined that RDX, HMX, TATP, and HMTD had unstable geometries in their reduced forms. The results should be useful in developing detection and screening methods including ionization methods for mass spectroscopy and fluorescence quenching methods of detection.

Keywords: ionization, affinity, explosive, TNT, ab initio, DFT

\section{Introduction}

Chemical screening and detection of common explosives in environments such as automobiles, airports, and mail have become the focus of intense research after events such as the 2001 attempt to destroy American Airlines Flight 63 from Paris in midflight using explosives hidden in a shoe. There have been many successful methods for explosives detection, ${ }^{1,2}$ which include pulse laser ionization time of flight, ${ }^{3,4}$ laser induced photofragmentation, ${ }^{5}$ ion mobility spectrometry, ${ }^{6,7}$ HPLC-diode array detection, ${ }^{8,9}$ electrochemical microfluidic device, ${ }^{10} \mathrm{LC} / \mathrm{MS}$-atmospheric pressure chemical ionization, ${ }^{11}$ and Raman. ${ }^{12}$ Other methods utilizing colorimetric ${ }^{13,14}$ detection (exposing a reagent to an explosive produces a color change) or fluorescence detection have been developed. Fluorescence techniques typically offer improved sensitivity. Fluorescence methods utilize a probe whose emission is quenched by the analyte. While these methods are not as selective as mass spectroscopy (MS) techniques, they offer rapid screening for use in the field. ${ }^{15,16}$ Several approaches for choosing the 
fluorescent probe have been studied. For instance, conjugated fluorescent polymers have been applied to the detection of gas phase 2,4,6-trinitrotoluene (TNT) ${ }^{17}$ and to a wide range of explosives in solution. ${ }^{18-21}$ Coupling of the fluorescent polymer to an orthogonal technique such as thin layer chromatography has proven effective in improving selectivity while maintaining low detection limits. ${ }^{22}$ Quantum dot (QD) fluorescent probes have successfully been applied to detecting TNT through use of amine capped $\mathrm{ZnS}: \mathrm{Mn}^{2+},{ }^{23} \mathrm{~L}$-cysteine capped $\mathrm{CdTe},{ }^{24}$ and $\mathrm{CdTe} / \mathrm{CdS}$ core/shell hybrid Au-nanorod assemblies. ${ }^{25}$ Detection limits as low as $5 \mathrm{ng} \mathrm{mm}^{-2}$ have been achieved on manila envelopes. ${ }^{26}$ However, the application of QD based techniques to a variety of explosives is limited.

Several studies suggest fluorescence quenching by a charge transfer mechanism where an excited electron in the conduction band (CB) of the probe (for example a conjugated polymer or quantum dot) is transferred to a lower lying acceptor state (likely the lowest unoccupied molecular orbital (LUMO) level) in the explosive where it can thereafter transfer back to the valence band (VB) of the probe. ${ }^{19,23,24}$ To help validate this model, an estimate of the vertical ionization potential $\left(\mathrm{IP}_{\mathrm{V}}\right)$ will help place the highest occupied molecular orbital (HOMO) level of the explosive with respect to the $\mathrm{CB}$ and VB energies. In addition, the electron affinity (EA) for the explosive analytes is needed to place the LUMO level with respect to the $\mathrm{VB}$ to determine if the reduction reaction is favorable. Sanchez et al calculated B3LYP/6-31G* level Kohn Sham HOMO and LUMO orbital eigenvalues for a number of explosives to explain quenching trends observed in conjugated fluorescent polymers. ${ }^{19}$ This knowledge may in turn aid in a better understanding of the fluorescence quenching mechanism and help expand methods to include a broader range of common and home-made explosives.

The calculated adiabatic ionization potential $\left(\mathrm{IP}_{\mathrm{A}}\right)$ can aid in optimization of ionization methods and techniques for MS detection and quantification. ${ }^{4}$ Some groups have focused on utilization of laser ionization rather than chemical or electron impact to generate analyte ions prior to MS analysis. ${ }^{3}$ The high energy needed to ionize these molecules using single photon absorption would necessitate the use of 100-150 nm laser light. In order to avoid the complications inherent in working in the deep UV, laser ionization is accomplished via non-linear absorption (for example two or three photon absorption) of a pulsed excitation laser. Optical paramagnetic amplifiers have provided tunable excitation lasers between $230 \mathrm{~nm}$ and $22 \mu \mathrm{m}$ in some cases, ${ }^{27,28}$ allowing for tunable excitation using a wavelength multiple of the ionization energy of the target analyte. As non-linear absorption is sensitive to input power density and is typically very inefficient for organic molecules, ${ }^{29}$ knowledge of the target ionization potential (IP) can allow for careful tuning of the excitation so as to optimize analyte ionization while minimizing background.

Six explosive compounds were studied in this report, including two nitroamines: hexogen (RDX) and octogen (HMX); two peroxide explosives: triacetone triperoxide (TATP) and hexamethylene triperoxide diamine (HMTD); as well as TNT and pentaerythritol tetranitrate (PETN). The molecular structures are provided in Figure 1. These classes of compounds offer an additional interesting test of current density functional theory (DFT) methods as the systems are highly correlated.

The accuracy of the calculated IP and EA is expected to vary depending both on the level of theory and basis set. These values were compared between several DFT methods including: B3LYP, ${ }^{30}$ CAM-B3LYP, ${ }^{31}$ and $\omega \mathrm{B} 97 \mathrm{XD} ;{ }^{32}$ the double hybrid method B2PLYPD, ${ }^{33}$ and the MP2 method. While B3LYP has been successfully applied in a wide range of applications including the prediction of heats of formation for a number of explosives ${ }^{34}$ and the decomposition reaction pathway of RDX and $\mathrm{HMX}^{35,36}$ as well as TATP, ${ }^{37}$ we wanted to test the success of two long-range corrected functionals in predicting IP and EA as well. CAM-B3LYP is the long range corrected version of B3LYP which uses 0.19 Hartree Fock (HF) and $0.81 \mathrm{~B} 88$ exchange for short range interactions and $0.65 \mathrm{HF}$ and $0.35 \mathrm{~B} 88$ at long range. ${ }^{31} \omega \mathrm{B} 97 \mathrm{XD}$ is a long range corrected function which uses $100 \% \mathrm{HF}$ exchange for long-range interactions whose cutoff is controlled by the value $\omega$ which was optimized by Chai et al. ${ }^{32}$ This functional
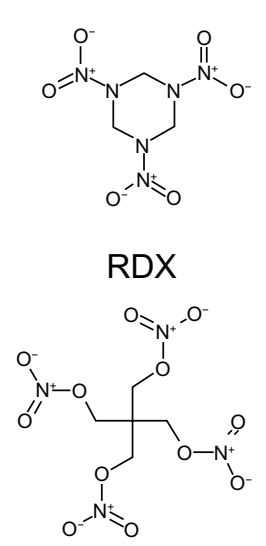

PETN

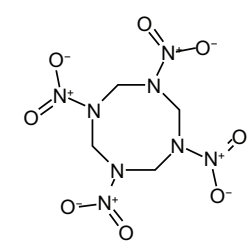

HMX

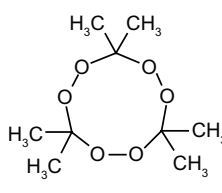

TATP

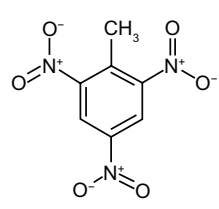

TNT

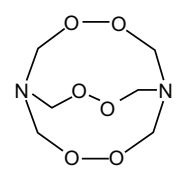

HMTD
Figure I Molecular structures of the six explosive compounds: RDX, HMX, TNT, PETN, TATP, and HMTD.

Abbreviations: RDX, hexogen; HMX, octogen; TNT, 2,4,6-trinitrotoluene; PETN, pentaerythritol tetranitrate; TATP, triacetone triperoxide; HMTD, hexamethylene triperoxide diamine. 
has an adjustable parameter $(\mathrm{X})$ to include short-range exact exchange while adding empirical dispersion correction (D). ${ }^{32}$ B2PLYPD is a double hybrid semi-empirical method that uses an optimized 0.53 mixing between HF and Becke (B) exchange while attempts to improve the correlation energy as obtained by (LYP) by applying a second-order correlation to the Kohn-Sham orbitals, like MP2, the contribution of which was optimized to 0.27 . This functional was also used with added empirical dispersion correction (D). ${ }^{33,38}$ Other mixed wave function and DFT double hybrid methods have proven successful in predicting IP and EA as compared to CBS-Q. ${ }^{39,40}$ Finally, these results were compared to the MP2 values. The methods were tested with three basis sets including 6-31G+(d,p), 6-311+G(d,p), and 6-311+G(3df,2p).

To provide a comparison for the above results, complete basis set method CBS-QB3 ${ }^{41}$ calculations were performed on each of the six compounds, as experimental data are unavailable in most cases. The CBS-QB3 method performs five successive calculations starting with geometry optimization and frequency calculation using B3LYP followed by three single point calculations using $\operatorname{CCSD}(\mathrm{T}), \mathrm{MP} 4 \mathrm{SDQ}$, and MP2 methods. The result can produce bond energies to within $1 \mathrm{kcal} / \mathrm{mol}(0.043 \mathrm{eV})$ accuracy. ${ }^{41}$ These calculations are excessively costly for these large systems; however, they provided a critical comparison method for the above described DFT and MP2 calculations. Discussion of the HOMO and LUMO molecular orbitals has not been addressed as it was not the direct focus of this work; however, detailed analysis of these and other orbitals of interest have been conducted and will be reported in detail in a separate study. The combination of DFT and wave function methods included in this study should provide a guide to future analytical efforts: it has been reported that DFT tends to overestimate the EA while HF methods underestimate it. ${ }^{42}$

\section{Computational methods}

All calculations were performed using the Gaussian $09^{43}$ program on a Sunfire X2200 M2 x64 server (2x Opteron quad core). Calculations were performed on geometry optimized structures in the gas phase. The geometries of the six common organic explosive molecules: RDX, $\beta$-HMX TATP, TNT, PETN, and HMTD were minimized using B3LYP/6-31+G(d,p) which has been reported to produce accurate ground state geometries. ${ }^{44}$ The minimized geometries were verified by calculating the vibrational energies to confirm there were no imaginary frequencies.

Single point energies were calculated on the geometry optimized structures for the neutral, cationic, and anionic form of each compound. The IP and EA were calculated from the single point energies using several methods including: B3LYP, CAM-B3LYP, $\omega B$ 97XD, MP2, and B2PLYPD. Three basis sets were used for each method which included $6-31+\mathrm{G}(\mathrm{d}, \mathrm{p}), 6-311+\mathrm{G}(\mathrm{d}, \mathrm{p})$, and 6-311+G(3df,2p). IP and EA were calculated from the absolute energies by using Equations 1 and 2, respectively, in which $\mathrm{M}, \mathrm{M}^{+}$, and $\mathrm{M}^{-}$, were the neutral, cationic, and anionic, forms of the optimized structures. ${ }^{45}$

$$
\begin{aligned}
& -I P=E\left(M^{+}\right)-E(M) \\
& -E A=E\left(M^{-}\right)-E(M)
\end{aligned}
$$

CBS-QB3 level calculations were performed for each of the compounds starting with the B3LYP/631+G(d,p) geometries described above. The IP and EA were taken from the HOMO and LUMO orbital energies, respectively. The ionized forms of TNT and RDX were calculated using CBS-QB3 by which the IP was calculated with Equation 1. These values are taken to be close to what would be measured experimentally for the EA and used as the reference comparisons and for calculating mean absolute deviation.

\section{Results and discussion \\ Total energy}

The minimized total energy $\left(\mathrm{E}_{\mathrm{h}}\right)$ for the six explosives (RDX, HMX, PETN, HMTD, TATP, and TNT) in the three charge states (neutral, cationic, and anionic) as calculated by the six methods tested (B3LYP, CAM-B3LYP, $\omega B$ 97XD, B2PLYPD, MP2, and CBS-QB3) using the 6-311+G(3df,2p) basis set is reported in Table 1. With the exception of the peroxides (TATP and HMTD), the remaining four explosives consistently had exothermic reduction potentials. As expected from the hybrid DFT methods (B3LYP, CAM-B3LYP, $\omega B$ 97XD, B2PLYPD), the minimized energy was well below the CBS-QB3 energy for each of the explosive molecules. This result is consistent with previous works that have concluded that methods like B3LYP are not variational. ${ }^{46}$ The double hybrid method, B2PLYPD, also reported a lower total energy than CBS-QB3; however, the magnitude of this result was less than in the single hybrid methods.

This effect is further emphasized in Figure 2 which shows the total energy $\left(\mathrm{E}_{\mathrm{h}}\right)$ for $\mathrm{RDX}(0), \mathrm{RDX}(+)$, and $\mathrm{RDX}(-)$ for all six methods and three basis sets tested. The trend reported in Figure 2 was the same for all the molecules studied. It is clear from this plot that B3LYP has the largest deviation from the CBS-QB3 energy while B2PLYPD has the least with CAMB3LYP and $\omega$ B97XD being essentially identical and between 
Table I Total energy (Hartree) of the six explosives studied by DFT methods, (B3LYP, CAM-B3LYP, and $\omega B$ 97XD) the double hybrid method (B2PLYPD), the wave function method (MP2), and the complete basis set method (CBS-QB3) for three charge states (neutral $(0)$, cationic $(I)$, and anionic $(-I))$ using the $6-3 I I+G(d, p)$ basis set

\begin{tabular}{|c|c|c|c|c|c|c|c|}
\hline Molecule & Charge & B3LYP & CAM-B3LYP & WB97XD & B2PLYPD & MP2 & CBS-QB3 \\
\hline \multirow[t]{3}{*}{ RDX } & 0 & -897.7363731 & -897.4104675 & -897.4492990 & -897.1493959 & -895.9971866 & -896.311576 \\
\hline & 1 & -897.3440172 & -896.9918423 & -897.0345343 & -896.7580594 & -895.6083858 & -895.944449 \\
\hline & -1 & -897.7801896 & -897.4402900 & -897.4707777 & -897.1651901 & -896.0219732 & \\
\hline \multirow[t]{3}{*}{ HMX } & 0 & || |96.988577| & -1196.5543026 & -1196.6114594 & -1196.2168147 & -1194.6819049 & -1195.0257560 \\
\hline & 1 & -1196.6086436 & -1196.1547082 & -1196.2160503 & -1195.8376596 & -1194.3084239 & \\
\hline & -1 & -1197.0370384 & $-1|96.592| 483$ & -1196.6412059 & -1196.2506587 & -1194.7087593 & \\
\hline \multirow[t]{3}{*}{ PETN } & 0 & -1316.9695950 & -1316.5055682 & $-|3| 6.543794 \mid$ & -1316.1340642 & -1314.4790096 & $-|3| 4.5 \mid 1793$ \\
\hline & 1 & $-13|6.564| 405$ & -1316.0678833 & -1316.1039710 & $-|3| 5.709997 \mid$ & $-|3| 4.064 \mid 528$ & \\
\hline & -1 & -1317.0236425 & -1316.5351929 & -1316.5625838 & -1316.1643149 & $-1314.4947 \mid 94$ & \\
\hline \multirow[t]{3}{*}{ HMTD } & 0 & -796.6869007 & -796.3779696 & -796.4325716 & -796.1280074 & -795.0421864 & -795.2729270 \\
\hline & 1 & -796.375305 I & -796.0497395 & -796.1090113 & -795.8130225 & -794.7156923 & \\
\hline & -1 & -796.6576837 & -796.3374652 & -796.3810768 & -796.0902273 & $-794.99707 / 8$ & \\
\hline \multirow[t]{3}{*}{ TATP } & 0 & -805.1801970 & -804.8350192 & -804.9224283 & -804.5796483 & -803.4364440 & -803.6523030 \\
\hline & 1 & -804.8644437 & -804.5007266 & -804.5934464 & -804.265338 I & -803.1299843 & \\
\hline & -1 & -805.1438201 & $-804.7890 \mid 22$ & $-804.86 \mid 5303$ & -804.5366606 & -803.3868315 & \\
\hline \multirow[t]{3}{*}{ TNT } & 0 & -885.3659690 & -885.0109916 & -885.0646472 & -884.7736948 & -883.5995416 & -883.9237220 \\
\hline & 1 & -884.9786462 & -884.6071939 & -884.6637417 & -884.3768578 & -883.1744860 & -883.5310470 \\
\hline & -1 & -885.4485079 & $-885.087 \mid 265$ & -885.1360175 & -884.8408476 & -883.6108086 & \\
\hline
\end{tabular}

Abbreviations: DFT, density functional theory; RDX, hexogen; HMX, octogen; PETN, pentaerythritol tetranitrate; HMTD, hexamethylene triperoxide diamine; TATP, triacetone triperoxide; TNT, 2,4,6-trinitrotoluene.

the B3PLYP and B2PLYPD energies. Also as expected, the MP2 energy was higher than the CBS-QB3 energy.

\section{Ionization potential}

For wave function based methods, the orbital eigenvalues correspond to measurable quantities where the HOMO and LUMO energies are the vertical IP ( $\left.\mathrm{IP}_{\mathrm{V}}\right)$ and EA $\left(\mathrm{EA}_{\mathrm{v}}\right)$, respectively, according to Koopmans' theory. This is not necessarily the case for DFT methods, as the Kohn-Sham HOMO orbitals are well known to not accurately reproduce IPs without corrections. ${ }^{45,47,48}$ However, comparisons of the $\mathrm{IP}_{\mathrm{V}}$ and $\mathrm{EA}_{\mathrm{V}}$ to the IP and EA as calculated by taking the difference between the cation and neutral molecule or anion and neutral molecule absolute energies, respectively, also known

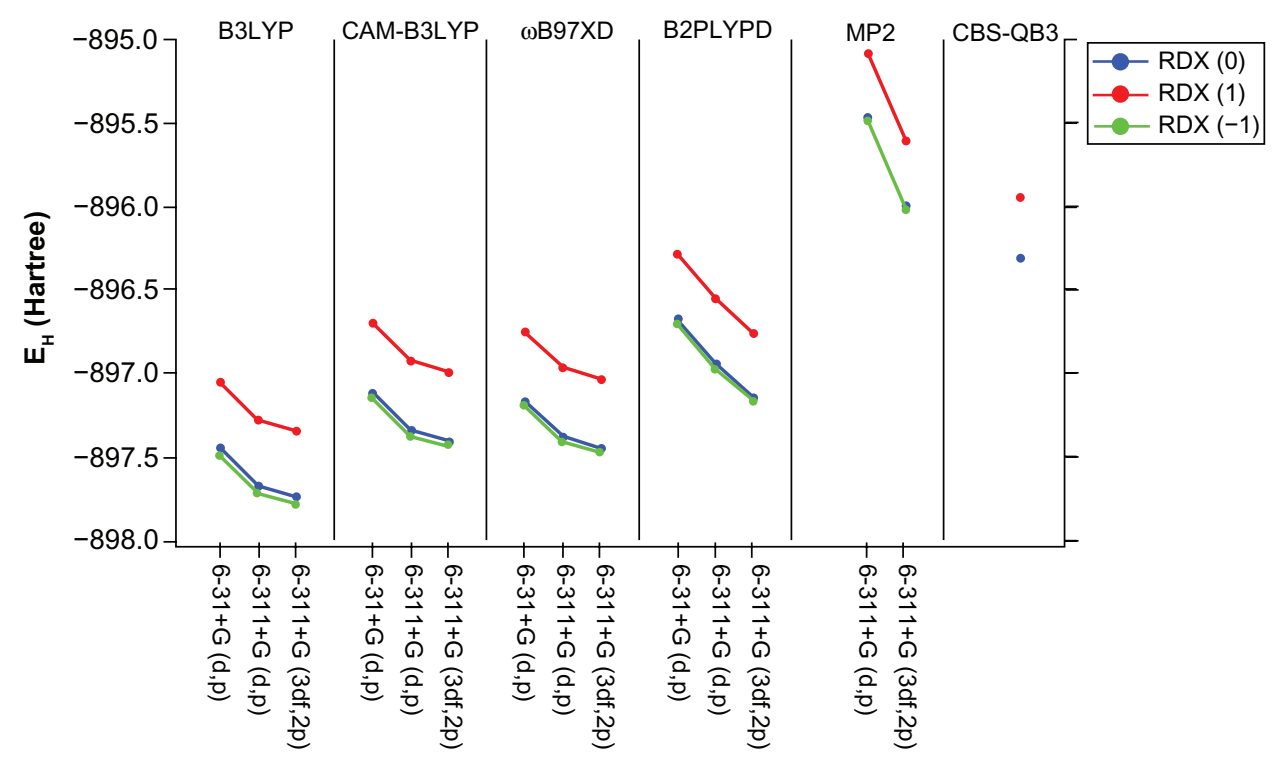

Figure 2 Plot of the total energy in Hartree for three charge states of RDX [neutral (blue), ionized (red), and reduced (green)] as a function of method (left to right: B3LYP, CAM-B3LYP, $\omega B$ 97XD, B2PLYPD, MP2, and CBS-QB3) for the three basis sets tested: $6-3 I+G(d, p), 6-3 \mid I+G(d, p)$, and 6-3II+G(3df,2p).

Abbreviations: RDX, hexogen; $E_{H}$, total energy. 
as the $\Delta \mathrm{E}$ method, often results in large differences ${ }^{49}$ with experimental data matching more closely the $\Delta \mathrm{E}$ method. ${ }^{44}$ Therefore, single point energies of the neutral, cation, and anion explosives were calculated for each of the explosives. An additional step may be taken to match experimental data more accurately in which a geometry optimization of the cation and anion is performed to calculate the adiabatic IP $\left(\mathrm{IP}_{\mathrm{A}}\right)$ and EA $\left(\mathrm{EA}_{\mathrm{A}}\right)$. This method was also applied to the compound set studied for the IP.

The IP as calculated by the $\Delta \mathrm{E}$ method (Equation 1 ) using B2PLYPD with three basis sets [6-31+G(d,p), 6-311+G(d,p), and $6-311+\mathrm{G}(3 \mathrm{df}, 2 \mathrm{p})]$ was compared to the Koopmans' theory method of calculating the IP by taking the HOMO orbital eigenvalue $(\varepsilon)$ as calculated by MP2 and CBS-QB3 (Figure 3). The $\Delta \mathrm{E}$ method more closely represents the $\mathrm{IP}_{\mathrm{A}}$ while the Koopmans' theory method is equivalent to the $\mathrm{IP}_{\mathrm{V}}$ There was a clear difference in the IP values calculated by Koopmans' theory and those calculated by the $\Delta \mathrm{E}$ method: the IPs were higher (between 0.77 and $3.04 \mathrm{eV}$ ) using Koopmans' theory. The MP2 6-311+G(3df,2p) level eigenvalues were very similar to those calculated by CBSQB3 making MP2 a much more cost effective approach. As has been previously mentioned, the $\Delta \mathrm{E}$ method generates IPs that more accurately represent experimentally determined values, which are typically equal to the $\mathrm{IP}_{\mathrm{A}}$ rather than the $\mathrm{IP}_{\mathrm{V}}$. The IP $\mathrm{I}_{\mathrm{V}}$ represents a Franck Condon transition from the vibrational ground state to ionization while not changing the nuclear coordinates while the $\mathrm{IP}_{\mathrm{A}}$ represents the energy

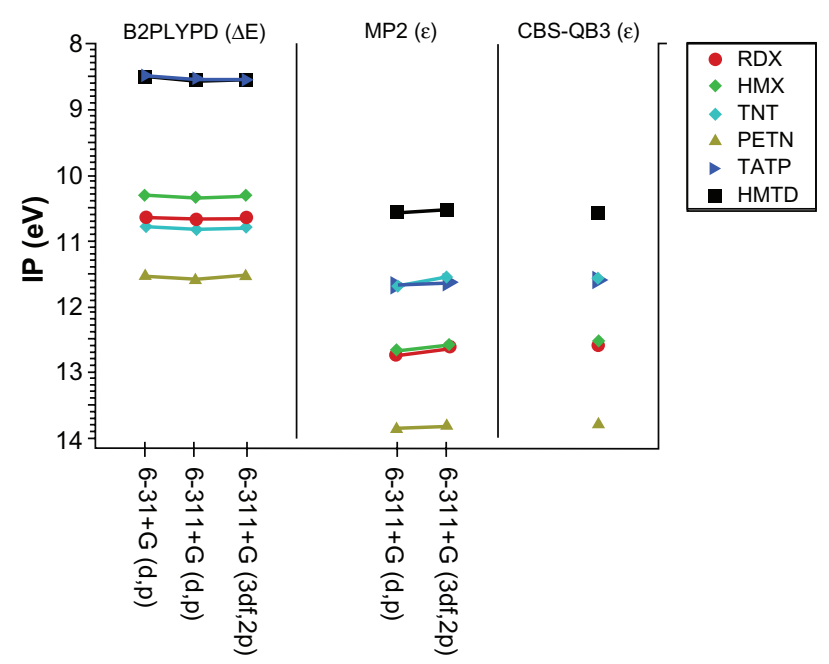

Figure 3 lonization potential of the six explosives: RDX (red), HMX (green), TNT (cyan), PETN (gold), TATP (blue), and HMTD (black) as calculated by B2PLYPD using the $\triangle \mathrm{E}$ method (Equation I), and the MP2 and CBS-QB3 orbital eigenvalues $(\varepsilon)$ using Koopmans' theory.

Abbreviations: RDX, hexogen; HMX, octogen; TNT, 2,4,6-trinitrotoluene; PETN, pentaerythritol tetranitrate; TATP, triacetone triperoxide; HMTD, hexamethylene triperoxide diamine; IP, ionization potential. difference between the vibrational ground states of the ion and neutral molecule. If the geometry of the ion does not change much from the neutral molecule, the IP and EA as calculated by the $\Delta \mathrm{E}$ method without optimization of the ion geometry can be quite close to the $\mathrm{IP}_{\mathrm{A}}$ after accounting for relaxation of the ion geometry. ${ }^{49}$ A major focus of this work is in predicting experimental IP; therefore, we concentrated on the $\Delta \mathrm{E}$ approach. However the $\mathrm{IP}_{\mathrm{V}}$ is expected to be an important factor in fluorescence quenching experiments when determining band alignment between QD or fluorescent polymers and acceptor levels in the explosives.

The IP for the six explosives was calculated using Equation 1 for the hybrid DFT methods as well as the MP2 method without geometry optimization of the ionized form. The $\mathrm{IP}_{\mathrm{A}}$ was calculated for two explosives (RDX and TNT) by CBS-QB3, however, the application of this method was strictly limited due to the tremendous cost of the calculations. The results are presented in Figure 4 for the six methods and three basis sets. The CBS-QB3 IPs are expected to be the experimental IPs that are applicable in electrochemistry studies as well as optimizing ionization methods for mass spectroscopy.

In general, CAM-B3LYP and $\omega$ B97XD both predicted IPs higher than that predicted by B3LYP and B2PLYPD. It is interesting to note that the IPs had little basis set dependence compared to the deviation between methods. The average standard deviation of the IPs within a DFT method between basis sets was $0.037 \mathrm{eV}$. The average standard deviation between methods for the same basis set was $1.25,1.23$, and $1.22 \mathrm{eV}$ for $6-31+\mathrm{G}(\mathrm{d}, \mathrm{p}), 6-311+\mathrm{G}(\mathrm{d}, \mathrm{p})$, and $6-311+\mathrm{G}(3 \mathrm{df}, 2 \mathrm{p})$, respectively. Therefore, the choice of method is more important than that of the basis set. The smallest basis set used was adequate over the more computationally costly ones.

With the exception of TNT, comparisons of the results to experimental data were not possible as experimental IPs for the explosives are not available, to the best of our knowledge. GC-MS techniques have been applied to approximate the IP for TNT by other groups. Langford et al reported the expected IP of TNT to be between $10.4 \mathrm{eV}$ and $13.4 \mathrm{eV},{ }^{50}$ Mullen et al reported a value below $10.49 \mathrm{eV},{ }^{4}$ while Potapov et al reported the IP as $10.59 \mathrm{eV} .^{51}$ For this reason, we calculated the $\mathrm{IP}_{\mathrm{A}}$ for TNT by CBS-QB3 which was found to be $10.685 \mathrm{eV}$. In this context, B3LYP (10.54 eV) and B2PLYPD (10.80 eV) outperformed CAM-B3LYP $(10.99 \mathrm{eV})$ and $\omega$ B97XD (10.91 eV). B2PLYPD is, however, much more costly than B3LYP. MP2 failed as the IP was calculated to be $11.57 \mathrm{eV}$ using the $\Delta \mathrm{E}$ method. 


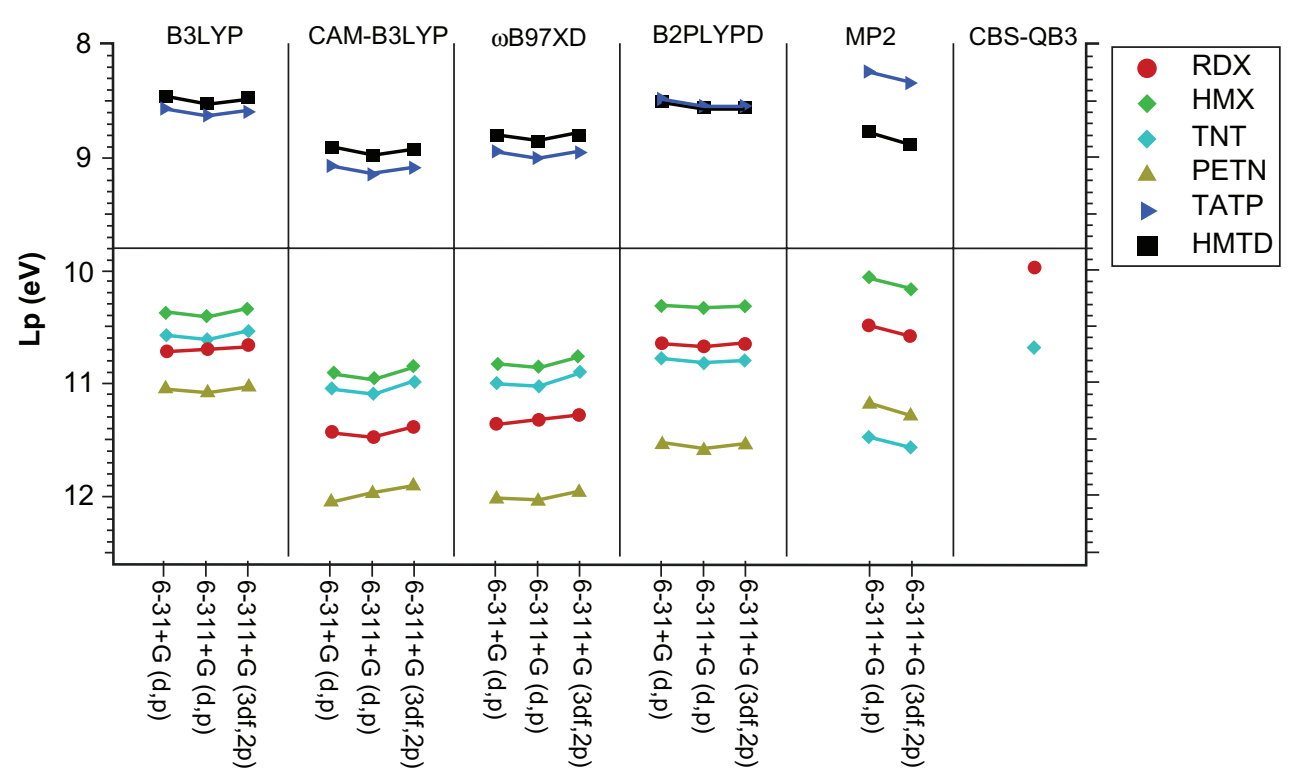

Figure 4 Plot of the ionization potential determined by Equation I ( $\triangle \mathrm{E}$ ) in eV of the six explosives: RDX (red), HMX (green), TNT (cyan), PETN (gold), TATP (blue), and HMTD (black); as calculated using (from left to right): B3LYP, CAM-B3LYP, $\omega B$ 97XD, B2PLYPD, MP2, and CBS-QB3 for the three basis sets 6-3I+G(d,p), 6-3II+G(d,p), and 6-3 I I+G(d,p). Adiabatic IP using CBS-QB3 was reported for RDX and TNT ( $\triangle E$ method).

Abbreviations: RDX, hexogen; HMX, octogen; TNT, 2,4,6-trinitrotoluene; PETN, pentaerythritol tetranitrate; TATP, triacetone triperoxide; HMTD, hexamethylene triperoxide diamine; IP, ionization potential.

Interestingly, B2PLYPD reproduced the adiabatic IP calculated using CBS-QB3 accurately.

The $\mathrm{IP}_{\mathrm{A}}$ was calculated as well by performing a geometry optimization on the cation with B3LYP and the $6-31+G(d, p)$ basis set followed by a single point energy calculation using B2PLYPD with the 6-31+G(d,p) basis set. The resulting IP for all the explosives was on average $0.25 \pm 0.03 \mathrm{eV}$ less (with the exception of RDX that was $0.50 \mathrm{eV}$ less) than the calculated IP without including the geometry optimization. If we take into account the stabilization energy from optimizing the cation geometry of TNT, the B2PLYPD predicted $\mathrm{IP}_{\mathrm{A}}$ was found to be $10.56 \mathrm{eV}$. This result makes B2PLYPD level calculations at least consistent with CBS-QB3 at a fraction of the cost.

TATP was reported by Mullen et al to have an expected IP between 9.4 and $9.7 \mathrm{eV}$ using pulsed laser ionization mass spectroscopy. ${ }^{3}$ These values were obtained by the assertions that the $9.4 \mathrm{eV}$ IP energy was the six photon energy of their laser $(795 \mathrm{~nm})$ which they used to cause ionization of TATP and the $9.7 \mathrm{eV}$ IP is that of acetone which the researchers used to bracket the expected IP. B3LYP and B2PLYPD calculations determined the IP to be $8.59 \mathrm{eV}$ and $8.55 \mathrm{eV}$, respectively, which is considerably lower than the expected result. However, it is not clear if this is due to inaccuracy of our calculations or if a $575 \mathrm{~nm}$ laser would also produce ionization through a four photon reaction. Further experimental evidence is required to validate our results.
The experimental IP for RDX could not be found in the literature. Our results from CBS-QB3 calculations found the $\mathrm{IP}_{\mathrm{A}}$ to be $9.99 \mathrm{eV}$. B3LYP and B2PLYPD calculated the IP to be 10.68 and $10.65 \mathrm{eV}$, respectively. If the geometry optimized cation was used, $\mathrm{IP}_{\mathrm{A}}$ calculated using B2PLYPD is $10.15 \mathrm{eV}$, which again is consistent with CBS-QB3. These results are consistent with the remaining explosives studied herein.

\section{Electron affinity}

It was the case for RDX, HMX, HMTD, and TATP that their molecular geometries were unstable when a geometry optimization was performed on the anionic form. For instance, HMTD in the reduced form was seen to break one of the oxygen to oxygen bonds. As a result, we have focused on the EA from the perspective of an equilibrium ground state geometry rather than attempting to make the same adiabatic correction which was made to the IP calculations. The EA for the six compounds, as calculated by Equation 2, was reported in Figure 5 for the hybrid DFT, double hybrid, and MP2 methods. The MP2 level calculation yielded orbital eigenvalues for the LUMO level which were inconsistent in magnitude and sign with those calculated using the other methods tested herein; however, the $\Delta \mathrm{E}$ method produced consistent and improved results for calculating the EA, The $\mathrm{EA}_{\mathrm{v}}$ was calculated using CBS-QB3 and was taken to be the negative of orbital eigenvalues of the LUMO state 


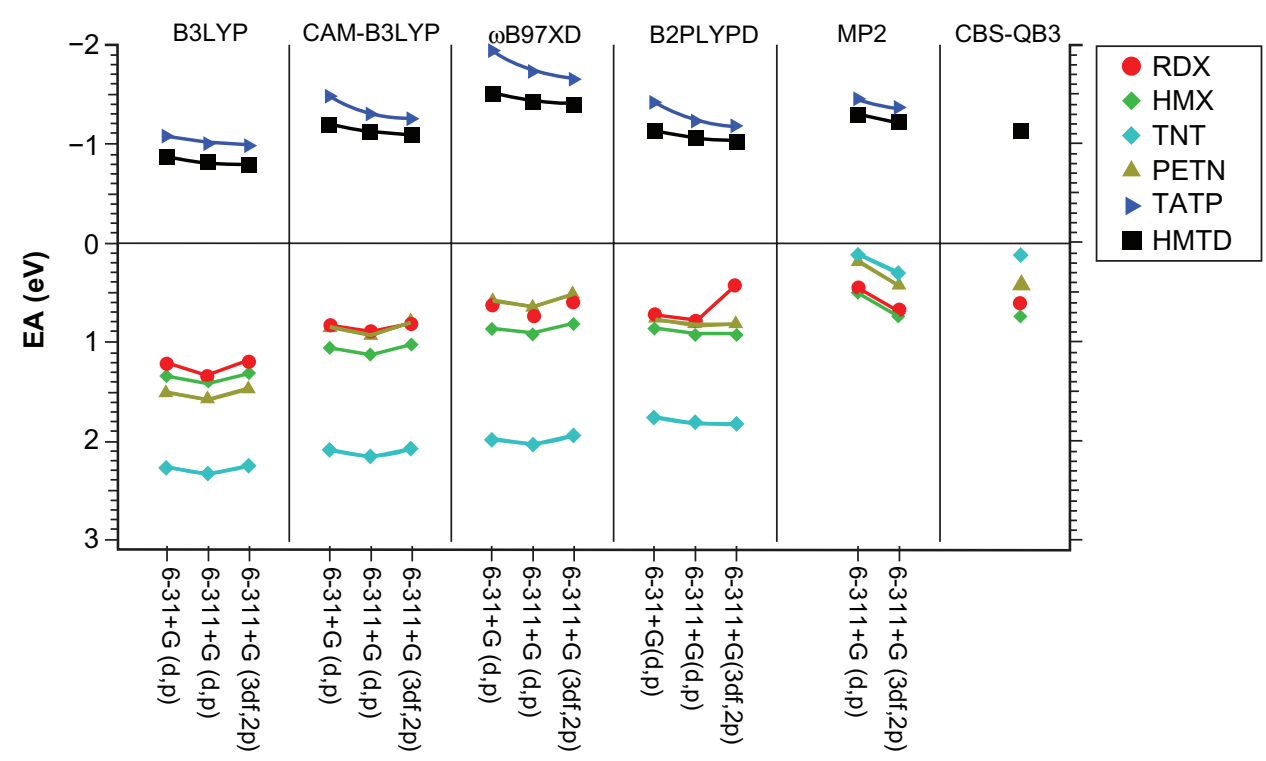

Figure 5 Electron affinities of the six explosives studied: RDX (red), HMX (green), TNT (cyan), PETN (gold), TATP (blue), and HMTD (black); as calculated by: B3LYP, CAM-B3LYP, $\omega B 97 X D, B 2 P L Y P D, M P 2$, and CBS-QB3; using the basis sets: 6-3I+G(d,p), 6-3II+G(d,p), and 6-3II+G(3df,2p).

Abbreviations: RDX, hexogen; HMX, octogen; TNT, 2,4,6-trinitrotoluene; PETN, pentaerythritol tetranitrate; TATP, triacetone triperoxide; HMTD, hexamethylene triperoxide diamine; EA, electron affinity.

using Koopmans' theory. These values were taken to be the expected experimental results and have henceforth been used to compare the accuracy of the other methods tested. A positive value of the EA indicates that the reduction reaction is exothermic. RDX, HMX, TNT, and PETN had positive reduction potentials as expected while the peroxides had negative values. The values ranged between 2.5 and $0.4 \mathrm{eV}$ for the former and -0.6 and $-2.0 \mathrm{eV}$ for the latter compounds. The EAs were very dependent on the computational method and to a lesser extent on the basis set.

Using CBS-QB3, the EAs for RDX, HMX, PETN, and TNT were found to be $0.61,0.70,0.40$, and $0.11 \mathrm{eV}$, respectively, while the EAs for TATP and HMTD were both found to be $-1.12 \mathrm{eV}$. There are no experimentally determined EAs of these compounds, with the exception of TNT which has been determined to be between $0.6-0.7 \mathrm{eV}^{52,53}$ The difference between the experimental EA for TNT and the one calculated herein could be a combination of experimental error (minimum of $0.2 \mathrm{eV}$ ) s3 $^{53}$ as well as an expected difference between the $\mathrm{EA}_{\checkmark}$ and $\mathrm{EA}_{\mathrm{A}}$. The $\mathrm{EA}_{\mathrm{A}}$ for TNT was approximated by a B3LYP geometry optimization and B2PLYPD $6-311+G(d, p)$ single point calculation of the anionic TNT which resulted in a correction of $0.16 \mathrm{eV}$ making the $\mathrm{EA}_{\mathrm{A}}$ for TNT approximately $0.27 \mathrm{eV}$.

It is clear from Figure 5 that the single and double hybrid DFT methods did not perform well on TNT. The error in the TNT EA was essentially consistent between the methods; however, it is not clear as to the source of such a deviation.
These methods did perform relatively well for the remaining unconjugated systems. $\omega$ B97XD had a larger deviation from the benchmark CBS-QB3 value for TATP and HMTD than the other methods tested though.

The mean average deviation was calculated with respect to the CBS-QB3 value and has been reported in Figure 6 . It is clear that while B3LYP performed well for predicting the IP, it performed the worst for EAs. CAM-B3LYP and $\omega \mathrm{B} 97 \mathrm{XD}$ were comparable but marked a large improvement over B3LYP while B2PLYPD outperformed the three. The EA as calculated by MP2 matched most closely with the

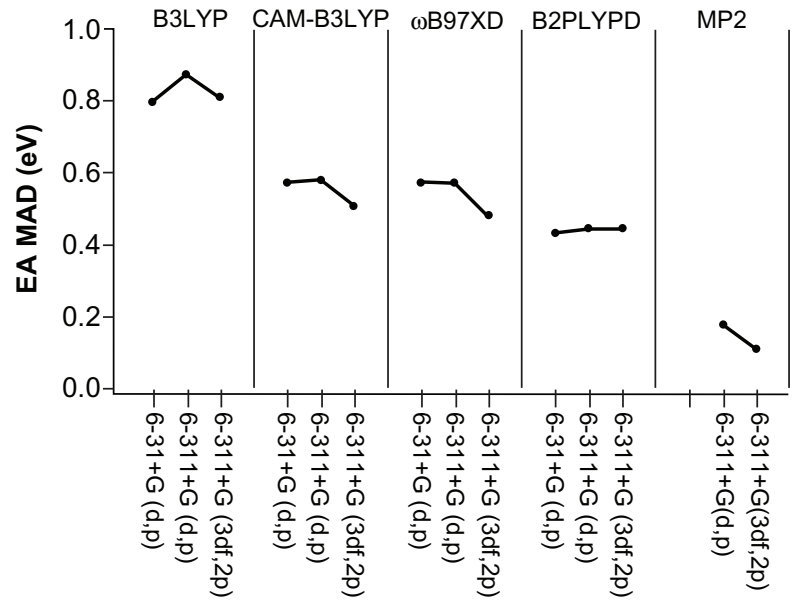

Figure 6 Electron affinity mean average deviation from the CBS-QB3 LUMO orbital eigenvalue as calculated by the B3LYP, CAM-B3LYP, $\omega B 97 X D, B 2 P L Y P D$, and MP2 methods using the $6-3 I+G(d, p), 6-3 \mid I+G(d, p)$, and $6-3 \mid I+G(d, p)$ basis sets.

Abbreviations: EA, electron affinity; MAD, mean average deviation. 
EA predicted by CBS-QB3 even though the former was calculated using the $\Delta \mathrm{E}$ method and the latter was from Koopmans' theory. As B2PLYPD is similar in cost to MP2, the next most cost effective method tested, by comparison, was $\omega \mathrm{B} 97 \mathrm{XD} / 6-311+\mathrm{G}(3 \mathrm{df}, 2 \mathrm{p})$.

\section{Conclusions}

Three hybrid DFT methods (B3LYP, CAM-B3LYP, and $\omega \mathrm{B} 97 \mathrm{XD})$, the double hybrid method B2PLYPD, and the wave function method MP2 were tested with three basis sets (6-31+G(d,p), 6-311+G(d,p), and 6-311+G(d,p)) along with a complete basis set method, CBS-QB3, in their application to predicting the IPs and EAs for six common explosive molecules. Calculations were performed on minimized neutral ground state geometries optimized by B3LYP as well as the geometry optimized ionized forms. The IP was found using both Koopmans' theory and the $\Delta \mathrm{E}$ method with the latter being representative of experimental evidence reported in previous works which were also consistent with the $\mathrm{IP}_{\mathrm{A}}$ calculated by CBS-QB3 for two test molecules. The predictive quality of the various methods depended on the property of interest (IP or EA), with B3LYP and B2PLYPD performing well for IP while MP2 and B2PLYPD performed the best for EAs. B2PLYPD consistently outperformed the other DFT methods; however, it is substantially more costly. The longrange correlated DFT functionals performed well for EAs but poorly for predicting IPs. The results should be useful in guiding further research in the field of explosives detection via fluorescence quenching and mass spectroscopy.

\section{Acknowledgments}

We would like to thank Professor Todd Wipke for his software resources and Professor Ilan Benjamin for providing the computational cluster. We would like to acknowledge useful discussions with Doctors Will Kuo and Riad Manna. Part of this work was performed under the auspices of the US Department of Energy by Lawrence Livermore National Laboratory under Contract DE-AC52-07 NA27344. JZZ is grateful to the BES Division of the US DOE and the NSF for financial support.

\section{Disclosure}

The authors report no conflicts of interest in this work.

\section{References}

1. Moore DS. Instrumentation for trace detection of high explosives. Rev Sci Instrum. 2004;75(8):2499.

2. Marshall M, Oxley JC. Aspects of explosives detection. The Netherlands: Elsevier; 2009.
3. Mullen C, Huestis D, Coggiola M, Oser H. Laser photoionization of triacetone triperoxide (TATP) by femtosecond and nanosecond laser pulses. Int J Mass Spectrom. 2006;252(1):69-72.

4. Mullen C, Irwin A, Pond BV, Huestis DL, Coggiola MJ, Oser H. Detection of explosives and explosives-related compounds by single photon laser ionization time-of-flight mass spectrometry. Anal Chem. 2006;78(11):3807-3814.

5. Cabalo J, Sausa R. Trace detection of explosives with low vapor emissions by laser surface photofragmentation-fragment detection spectroscopy with an improved ionization probe. Appl Opt. 2005;44(6):1084-1091.

6. Oxley JC, Smith JL, Kirschenbaum LJ, Marimganti S, Vadlamannati S. Detection of explosives in hair using ion mobility spectrometry. J Forensic Sci. 2008;53(3):690-693.

7. Creaser CS, Griffiths JR, Bramwell CJ, Noreen S, Hill CA, Thomas CLP. Ion mobility spectrometry: a review. Part 1 . Structural analysis by mobility measurement. Analyst. 2004;129(11):984-994.

8. Borch T, Gerlach R. Use of reversed-phase high-performance liquid chromatography-diode array detection for complete separation of 2 , 4,6-trinitrotoluene metabolites and EPA Method 8330 explosives: influence of temperature and an ion-pair reagent. $J$ Chromatogr A. 2004;1022(1):83-94.

9. Schulte-Ladbeck R, Kolla P, Karst U. Trace Analysis of Peroxide-Based Explosives. Anal Chem. 2003;75(4):731-735.

10. Hilmi A, Luong JH. Electrochemical detectors prepared by electroless deposition for microfabricated electrophoresis chips. Anal Chem. 2000;72(19):4677-4682.

11. Crowson A, Beardah MS. Development of an LC/MS method for the trace analysis of hexamethylenetriperoxidediamine (HMTD). Analyst. 2001;126(10):1689-1693.

12. Tuschel DD, Mikhonin AV, Lemoff BE, Asher SA. Deep Ultraviolet Resonance Raman Excitation Enables Explosives Detection. Appl Spectrosc. 2010;64(4):425-432.

13. Lin H, Suslick KS. A Colorimetric Sensor Array for Detection of Triacetone Triperoxide Vapor. J Am Chem Soc. 2010;132(44):15519-15521.

14. Reynolds J, Nunes P, Whipple R, Alcaraz A. On-site analysis of explosives in various matrices. In: Schubert H, Kuznetsov A, editors. Detection and Disposal of Improvised Explosives. The Netherlands: Springer; 2006:27-32.

15. Schulte-Ladbeck R, Kolla P, Karst U. A field test for the detection of peroxide-based explosives. Analyst. 2002;127(9):1152-1154.

16. Girotti S, Ferri E, Maiolini E, et al. A quantitative chemiluminescent assay for analysis of peroxide-based explosives. Anal Bioanal Chem. 2011;400(2):313-320.

17. McQuade DT, Pullen AE, Swager TM. Conjugated polymer-based chemical sensors. Chem Rev. 2000;100(7):2537-2574.

18. Sanchez JC, Trogler WC. Efficient blue-emitting silafluorenefluorene-conjugated copolymers: selective turn-off/turn-on detection of explosives. J Mater Chem. 2008;18(26):3143-3156.

19. Sanchez JC, DiPasquale AG, Rheingold AL, Trogler WC. Synthesis, Luminescence Properties, and Explosives Sensing with 1,1-Tetraphenylsilole-and 1,1-Silafluorene-vinylene Polymers. Chem Mater. 2007;19(26):6459-6470.

20. Toal SJ, Magde D, Trogler WC. Luminescent oligo (tetraphenyl) silole nanoparticles as chemical sensors for aqueous TNT. Chem Commun (Camb). 2005;(43):5465-5467.

21. Sohn H, Sailor MJ, Magde D, Trogler WC. Detection of nitroaromatic explosives based on photoluminescent polymers containing metalloles. J Am Chem Soc. 2003;125(13):3821-3830.

22. Martinez HP, Grant CD, Reynolds JG, Trogler WC. Silica anchored fluorescent organosilicon polymers for explosives separation and detection. J Mater Chem. 2012;22(7):2908-2914.

23. Tu R, Liu B, Wang Z, et al. Amine-capped ZnS-Mn2+ nanocrystals for fluorescence detection of trace TNT explosive. Anal Chem. 2008;80(9): 3458-3465.

24. Chen Y, Chen Z, He Y, et al. L-cysteine-capped CdTe QD-based sensor for simple and selective detection of trinitrotoluene. Nanotechnology. 2010;21(12):125502. 
25. Xia Y, Song L, Zhu C. Turn-On and Near-Infrared Fluorescent Sensing for 2,4,6-Trinitrotoluene Based on Hybrid (Gold Nanorod)-(Quantum Dots) Assembly. Anal Chem. 2011;83(4):1401-1407.

26. Zhang K, Zhou H, Mei Q, et al. Instant Visual Detection of Trinitrotoluene Particulates on Various Surfaces by Ratiometric Fluorescence of Dual-Emission Quantum Dots Hybrid. J Am Chem Soc. 2011; 133(22):8424-8427.

27. Newhouse RJ, Wang H, Hensel JK, Wheeler DA, Zou S, Zhang JZ. Coherent Vibrational Oscillations of Hollow Gold Nanospheres. JPhys Chem Lett. 2011;2(3):228-235.

28. Cooper JK, Ling Y, Longo C, Li Y, Zhang JZ. Effects of Hydrogen Treatment and Air Annealing on Ultrafast Charge Carrier Dynamics in $\mathrm{ZnO}$ Nanowires Under in Situ Photoelectrochemical Conditions. J Phys Chem C. 2012;116(33):17360-17368.

29. Strehmel B, Sarker AM, Detert H. The influence of sigma and pi acceptors on two-photon absorption and solvatochromism of dipolar and quadrupolar unsaturated organic compounds. Chemphyschem. 2003;4(3):249-259.

30. Becke AD. Density-functional thermochemistry. IV. A new dynamical correlation functional and implications for exact-exchange mixing. J Chem Phys. 1996;104(3):1040-1046.

31. Yanai T, Tew DP, Handy NC. A new hybrid exchange-correlation functional using the Coulomb-attenuating method (CAM-B3LYP). Chem Phys Lett. 2004;393(1-3):51-57.

32. Chai JD, Head-Gordon M. Long-range corrected hybrid density functionals with damped atom-atom dispersion corrections. Phys Chem Chem Phys. 2008;10(44):6615-6620.

33. Grimme S. Semiempirical hybrid density functional with perturbative second-order correlation. J Chem Phys. 2006;124(3):034108.

34. Byrd EF, Rice BM. Improved Prediction of Heats of Formation of Energetic Materials Using Quantum Mechanical Calculations. J Phys Chem A. 2006;110(3):1005-1013.

35. Chakraborty D, Muller RP, Dasgupta S, Goddard WA. A detailed model for the decomposition of nitramines: RDX and HMX. Journal of Computer-Aided Materials Design. 2001;8(2):203-212.

36. Chakraborty D, Muller RP, Dasgupta S, Goddard III WA. Mechanism for unimolecular decomposition of $\operatorname{HMX}(1,3,5,7$-tetranitro-1, 3,5,7-tetrazocine), an ab initio study. J Phys Chem A. 2001;105(8): 1302-1314.

37. Dubnikova F, Kosloff R, Almog J, et al. Decomposition of triacetone triperoxide is an entropic explosion. J Am Chem Soc. 2005;127(4): 1146-1159.

38. Schwabe T, Grimme S. Double-hybrid density functionals with long-range dispersion corrections: higher accuracy and extended applicability. Phys Chem Chem Phys. 2007;9(26):3397-3406.
39. Zhao Y, Lynch BJ, Truhlar DG. Multi-coefficient extrapolated density functional theory for thermochemistry and thermochemical kinetics. Phys Chem Chem Phys. 2005;7(1):43-52.

40. Zhao Y, Lynch BJ, Truhlar DG. Doubly hybrid meta DFT: New multi-coefficient correlation and density functional methods for thermochemistry and thermochemical kinetics. J Phys Chem A. 2004;108(21):4786-4791.

41. Montgomery JA, Frisch MJ, Ochterski JW, Petersson GA. A complete basis set model chemistry. VI. Use of density functional geometries and frequencies. J Chem Phys. 1999;110(6):2822-2827.

42. Wroblewski T, Hubisz K, Antonowicz J. Theoretical study of electron affinities for selected diatomic molecules. Optica Applicata. 2010;40(3):601-608.

43. Frisch MJ, Trucks GW, Schlegel HB, et al. Gaussian 09. 2009.

44. Lewars EG. Computational Chemistry: Introduction to the Theory and Applications of Molecular and Quantum Mechanics. 2nd ed. New York: Springer; 2011.

45. Zhan C-G, Nichols JA, Dixon DA. Ionization Potential, Electron Affinity, Electronegativity, Hardness, and Electron Excitation Energy: Molecular Properties from Density Functional Theory Orbital Energies. J Phys Chem A. 2003;107(20):4184-4195.

46. Amovilli C, March NH, Bogár F, Gál T. Use of ab initio methods to classify four existing energy density functionals according to their possible variational validity. Phys Lett A. 2009;373(35):3158-3160.

47. Zhang G, Musgrave CB. Comparison of DFT Methods for Molecular Orbital Eigenvalue Calculations. J Phys Chem A. 2007;111(8): 1554-1561.

48. Stowasser R, Hoffmann R. What do the Kohn-Sham orbitals and eigenvalues mean? J Am Chem Soc. 1999;121(14):3414-3420.

49. Ghosh D, Golan A, Takahashi LK, Krylov AI, Ahmed M. A VUV Photoionization and $\mathrm{Ab}$ Initio Determination of the Ionization Energy of a Gas Phase Sugar (Deoxyribose). J Phys Chem Lett. 2012;3(1):97-101.

50. Langford ML, Todd JFJ. Negative-ion fragmentation pathways in 2,4,6-trinitrotoluene. Org Mass Spectrom. 1993;28(7):773-779.

51. Potapov VK, Kardash IE, Sorokin VV, Sokolov SA, Evlasheva TI. Photoionization of Heteroaromatic Compounds. Khim. Vys. Energ. 1972; 6:392.

52. Foster R. Organic Charge-Transfer Complexes. New York: Academic Press; 1969.

53. Briegleb G. Electron Affinity of Organic Molecules. Angew Chem Int Ed Engl. 1964;3(9):617-632.
Reports in Theoretical Chemistry

\section{Publish your work in this journal}

Reports in Theoretical Chemistry is an international, peer-reviewed, open access journal publishing original research, reports, reviews and commentaries on all areas of theoretical chemistry. The manuscript management system is completely online and includes a very quick and fair

\section{Dovepress}

peer-review system. Visit http://www.dovepress.com/testimonials.php to read real quotes from published authors. 